\title{
Application of Correlation Analysis in Conventional Plant Breeding and Genome Wide Association Mapping
}

\author{
Praveen Kumar $^{1 *}$ and Mainu Hazarika ${ }^{2}$ \\ ${ }^{1}$ Lovely Professional University-Phagwara (Punjab), India \\ ${ }^{2}$ Assam Agricultural University-Jorhat (Assam), India \\ *Corresponding author
}

\section{A B S T R A C T}

\section{Keywords}

Conventional Plant Breeding, Genome Wide Association Mapping

\section{Article Info}

Accepted:

26 July 2020

Available Online:

10 August 2020
Information on correlation of yield and its component attributes and their direct and indirect effects on grain yield are of paramount significance. Correlation in combination with path analysis would give a better insight into cause and effect relationship between different pairs of characters. It is also helpful to study the correlation in genome wide association mapping to deep insights the linkage, direction and magnitude of each and every traits of grain yield and its components traits.

\section{Introduction}

A genetic association is the proportion of variance that two properties share in multivariate quantitative genetics owing to genetic factors (Falconer, 1960), Association of genetic trait influences with genetic trait influences (Matin and Eaves, 1977), measuring the degree of pleiotropy or causal similarity. A genetic correlation of zero means that there is no association between the traits and one one morphological trait or gene does not affect the second morphological trait or gene and they are having a separate effect of them, while a correlation value is one, it is indicated that all the genetic factors on all traits are equal and correlation is very good between them. Models of genetic association were incorporated into behavioral genetics during the 1970s-1980s. Models of genetic association were developed in behavior genetics in the 1970s-1980s. Correlation tests the relationship between various plant characters and decides the components on which to base selection for improvement. Awareness of the correlation between key characters will foster proper tests analysis and to provide a framework for preparing more successful crop improvement programs. Phenotypic similarity refers to the degree of the association between two characters that has been observed. In comparison, the 
intrinsic connection between characters is the genotypical similarity. Knowledge of the interrelationship of various yield components is of utmost importance to a plant breeder when deciding on selection criteria. Correlation is very important for selection the all the component traits, which are contributing toward the yield (grain yield for the cereals, and pulses; oil content for the soybean, sesamum, groundnut etc.; fodder yield for the fodder sorghum, and fodder barley etc.). Correlation is using in the path analysis, discriminant analysis foe selection the important traits, also for the analysis the morphological diversity or molecular diversity. Correlation is also using the increasing the genetic gain using the different model of genomic selection. Genetic gain means is increasing mean value of progeny over the parental mean value, and it depend upon the selection intensity, phenotypic standard deviation, heritability and selection cycle per generation. Genetic associations have implications in genome-wide association analysis (GWAS) testing tests, reproduction, phenotype assessment and exploration of phenotype etiology \& disease. Twin research and molecular genetics may be used to predict these.

Genetic similarities were shown to be normal in plant genetics and to be largely close to their corresponding phenotypic correlations, and were also identified broadly in plant characteristics, called the 'phenome' (Falconer, 1960). A genetic association is to be associated with an environmental association between the two-trait conditions (e.g. that insufficient nutrition in a plant population causes both lower yields and plant height); a genetic association between two traits which contributes to the measurable association between these two traits, but genetic associations may also be the contradictory of phenotypic associations identified.

\section{Discussion of correlation in plant breeding}

The values of genetic correlations are different, its due to the heritability of every trait is not same, and they refer to the total additive variance component from the total phenotypic variance of the two sets of traits; the heritability of these two traits are high but they are may not be genetically associated or we can say they have very low heritability and be the entire correlated (as long as the heritability is not zero). Suppose in a population of maize (Zea mays L.), there are two characteristics -red seed and maize protein content. These two characters may have a very high heritability and they are independent from each other. Means variability in the population is because of these two traits. And its also helpful to identifying the meta QTLs (QTLS, which are contributing maximum phenotypic variability in the population) but they would also have a very less genetic association between these two traits.

\section{The causes of genetic correlations are following}

Linkage Disequilibrium (LD): they are transferred together or we say they in haplotype in chromosome, and they are producing the different phenotype in population

Pleiotropy (When a gene affecting the many phenotypes, eg. QPM varieties in maize

Mediated pleiotropy (when a gene produce a phenotype $X$ and this phenotype $X$ affect the second phenotyps gene and produce another trait that is Y).

Biases: assortative mating: "mating among the individual which are phenotypically or genotypically similar to each other). 


\section{Uses}

\section{Causes of changes in traits}

Genetic associations are clinically beneficial as hereditary similarities can be examined longitudinally through time (e.g., Hewitt et al., 1988). Yellow colour of maize seed would transfer to generation to subsequent generation.

\section{Boosting genome wide association mapping to accelerate the genetic gain}

\section{Genome-wide selection}

It is another approach that can be used to transfer the all favorable alleles for minor effect quantitative loci at whole genomic level (Meuwissen et al., 2001). In genome wide association selection, calculate the effect of each minor allele by using the genetic correlation or this can be analyze by genomewide effects for one trait, which can we easily measure or record the data, to increase the previous chance of variations for a second or another trait; because genetic gain and phenotypes are highly genetically correlated agenome wide association study for increasing crop yield will transfer also be a GWAS for genetic gain in crop yield and will be able to predicting variation in genetic gain as well as the best single nucleotide polymorphism candidates can be used to improve the statistical potential of a minor GWAS, a hybrid latent trait study analysis done where each determined geneticallycorrelated trait tends to minimize measuring error and considerably enhances the strength of GWAS (Krapohl et al., 2017).

Genetic associations may even calculate the contribution of similarities $<1$ through databases that may generate a misleading "lost heritage" through calculating to what degree various methods of measuring, ethnic factors, or conditions produce even partly related sets of appropriate genetic variants.

\section{Breeding}

The application of genetic correlations is also very valuable in applications such as conventional plant breeding and animal breeding by allowing to identified all the very useful characteristics, which is contributing toward the grain yield or milk production (especially for taking those character, which are sex-related or binary characteristics). Threshold model, where phenotype variations can not often be found but another strongly correlated trait, probably in behavioral phenotypes process in all plants, compensate for the various features than breeding, allowing breeding success predictions more accurate using the multivariate analysis in plant breeding which is can compared to predict based on the univariate equation of plant breeder using the multivariate breeder's equation, only per-heritability and allowing equality of characteristics, and eliminating unforeseen effects taking into justification the selection by artificial manner for or against trait $\mathrm{X}$ will also increasing or decreasing effects of all characteristics the positive direction or negative direction apply to $\mathrm{X}$ (Lerner 1950 and Falconer 1960). The selection limits imposed by trait intercorrelation, and the probability of genetic associations to alter over long-term breeding programmes.

In conclusion the correlation is an important statistical tool to identifying the all-important traits which are contributing towards the yield. It will boost the grain yield, because its applied in the genomic selection strategies.

\section{References}

Falconer 1960, Introduction to Quantitative Genetics, Ch. $19 \quad$ "Correlated 
Characters"

Lerner 1950, Population genetics and animal improvement: as illustrated by the inheritance of egg production

Krapohl et al., 2017, "Multi-polygenic score approach to trait prediction"

Hill et al., 2017, "A combined analysis of genetically correlated traits identifies 107 loci associated with intelligence"

Deary et al., 2012, "Genetic contributions to stability and change in intelligence from childhood to old age"
Hewitt et al., 1988, "Resolving the causes of developmental continuity or 'tracking.' I. Longitudinal twin studies during growth.

Pickrell 2015, "Fulfilling the promise of Mendelian randomization

Martin \& Eaves 1977, "The Genetical Analysis of Covariance Structure"

Krapohl et al., 2015, "Phenome-wide analysis of genome-wide polygenic scores"

\section{How to cite this article:}

Praveen Kumar and Mainu Hazarika. 2020. Application of Correlation Analysis in Conventional Plant Breeding and Genome Wide Association Mapping. Int.J.Curr.Microbiol.App.Sci. 9(08): 3372-3375. doi: https://doi.org/10.20546/ijcmas.2020.908.389 